\title{
Structural Synthon Approach to Predict the Possible Polytypes of Layered Double Hydroxides
}

\author{
S. Radha ${ }^{[a]}$ and P. Vishnu Kamath*[a]
}

Keywords: Structural synthons; Polytypes; Disorder; Layered double hydroxides; Zinc; Aluminum

\begin{abstract}
The complete universe of possible polytypes of layered double hydroxides (LDH) is predicted on the basis of symmetry arguments. A single $\left[\mathrm{MX}_{2}\right](\mathrm{X}=\mathrm{OH})$ layer, also defined as a structural synthon, belongs to the layer group $P \overline{3} 2 / m 1$. These layers can be stacked in such a way as to conserve the unique 3 -axis of the layer in the resultant crystal. The different stacking sequences that facilitate symmetry conservation, yield the different possible polytypes of rhombohedral and hexagonal symmetries. More polytypes can be envisaged by including stacking sequences that systematically destroy the princi-
\end{abstract}

pal symmetry elements of the structural synthon. Thereby, stacking sequences that destroy the 3 -axis, while retaining the 2-axis, yield possible polytypes of monoclinic symmetry. The nitrate-containing LDH of zinc and aluminum crystallizes in a faulted structure in which, the planar faults are shown to arise on account of stacking sequences whose local symmetry is monoclinic. This approach to polytype prediction expands on an earlier reported method by Bookin and Drits and is very general with important implications for other classes of layered materials.

\section{Introduction}

Layered materials are characterized by strong iono-covalent bonding along two dimensions and weak bonding along the third dimension, which is also the stacking direction. Given the weak bonding between layers, the layers can stack, in a multiplicity of patterns, called stacking sequences, to yield different polytypes. ${ }^{[1]}$ Polytypism is best defined as polymorphism in one dimension. ${ }^{[2]}$ Among the layered materials, layered double hydroxides (LDHs), have received a great deal of attention, on account of their applications in catalysis, sorption, anion exchange, water purification and in medicine, as agents for drug delivery. ${ }^{[3-6]}$

The structure of the LDHs is derived from that of mineral brucite, $\mathrm{Mg}(\mathrm{OH})_{2} \cdot{ }^{[7]}$ Brucite comprises a hexagonal packing of $\mathrm{OH}^{-}$ions in which $\mathrm{Mg}^{2+}$ ions occupy alternative layers of octahedral sites. This leads to a stacking of charge neutral layers having the composition $\left[\mathrm{Mg}(\mathrm{OH})_{2}\right]$. Bookin and Drits, ${ }^{[8]}$ represent such a layer by the symbol $\mathrm{AbC}$, by making use of the notation used to describe the close packing of atoms. Here the uppercase symbols A, B, C represent hydroxyl ion positions and lower case symbols represent cation positions in octahedral interstitials. Since the cation positions are determined by the hydroxyl ion positions, the layer can be more simply described by the symbol AC. The A, B and C positions are

\footnotetext{
* Prof. Dr. P. V. Kamath

E-mail: vishnukamath8@hotmail.com

[a] Department of Chemistry

Central College

Bangalore University

Bangalore-5600 01, India

Supporting information for this article is available on the WWW under http://dx.doi.org/10.1002/zaac.201200261 or from the author.
}

related to one another by successive translations of $(1 / 3,2 / 3)$ in the $a, b$ plane.

When a fraction $x$ of $\mathrm{Mg}^{2+}$ ions is isomorphously substituted by a trivalent ion such as $\mathrm{Al}^{3+}$, the metal hydroxide layers acquire the composition $\left[\mathrm{Mg}_{1-x} \mathrm{Al}_{x}(\mathrm{OH})_{2}\right]^{x+}$ and develop a positive charge, but their structure remains unchanged and each layer is still represented by the symbol AC. The resulting compound now incorporates anions and water molecules in the interlayer region to restore charge neutrality. ${ }^{[9]}$

Bookin and Drits predicted all the polytypes that can be obtained in theory by the stacking of AC layers. ${ }^{[8,10]}$ The different stacking sequences were then classified according to the crystal symmetry and symmetry of the interlayer sites. For instance, the stacking sequence

$\mathrm{AC}-\mathrm{AC}-\mathrm{AC} \ldots \ldots 1 \mathrm{H}$

is given the symbol $1 \mathrm{H}$, to describe a one layer polytype of hexagonal symmetry and '-' denotes an octahedral interlayer site. All two layer polytypes have hexagonal symmetry:

$\mathrm{AC}=\mathrm{CA}=\mathrm{AC}=\mathrm{CA} \ldots \ldots 2 \mathrm{H}_{1}$

$\mathrm{AC}-\mathrm{AB}-\mathrm{AC}-\mathrm{AB} \ldots \ldots .2 \mathrm{H}_{2}$

$\mathrm{AC}=\mathrm{CB}-\mathrm{AC}=\mathrm{CB} \ldots \ldots 2 \mathrm{H}_{3}$.

Here ' $=$ ' denotes a prismatic interlayer site. Among the three-layer polytypes, two belong to rhombohedral crystal symmetry and seven to hexagonal symmetry.

The Bookin and Drits scheme of classifications has had a fair degree of success in accounting for the structures of both mineral and synthetic LDHs. ${ }^{[10]}$ Carbonate containing minerals hydrotalcite and manessite have the structures of the $3 R_{1}$ and $2 \mathrm{H}_{1}$ polytypes respectively. ${ }^{[11]}$ The sulfate containing minerals have the structures of the $1 \mathrm{H}, 2 \mathrm{H}_{1}$ and $3 \mathrm{R}_{1}$ polytypes. ${ }^{[12]}$ Laboratory-synthesized sulfate LDHs crystallize in the structure of $1 \mathrm{H}$ and $3 \mathrm{R}_{1}$ polytypes depending on the precipitation conditions and exhibit solid-solid interpolytype transformations as a 
function of temperature and relative humidity. ${ }^{[13,14]}$ In many instances, laboratory-synthesized LDHs crystallize with the incorporation of a very large number of stacking faults, which can indeed be interpreted in terms of the intergrowths of more than one polytypes. ${ }^{[15]}$ Thus, the $\mathrm{Mg} / \mathrm{Al}, \mathrm{Co} / \mathrm{Fe}$ and $\mathrm{Ni} / \mathrm{Al}$ LDHs have been shown to be random intergrowths of the $3 \mathrm{R}_{1}$ and $2 \mathrm{H}_{1}$ polytypes. ${ }^{[16,17]}$

The nitrate containing LDHs have remained an enigma.

1. The nitrate LDHs exhibit two different interlayer spacings: $8.8 \AA(x=0.33)$ and $8.0 \AA(x=0.25) .^{[18]}$

2. This difference in the interlayer spacings is associated with the orientation of the $\mathrm{NO}_{3}{ }^{-}$ion in the interlayer. ${ }^{[19]}$ In the $\mathrm{LDH}$ with higher layer charge $(x=0.33)$, the $\mathrm{NO}_{3}{ }^{-}$ion intercalates with one of its $C_{2}$ axes parallel to the stacking direction (coordination symmetry, $C_{2 v}$ ). When the layer charge is lower, the $\mathrm{NO}_{3}{ }^{-}$ion intercalates with its molecular plane perpendicular to the stacking direction (coordination symmetry, $D_{3 h}$ ).

3. Nitrate containing LDHs are poorly ordered and no reliable structure models are available in the literature. As we shall show later in this paper, the powder diffraction profile of the $\mathrm{NO}_{3}{ }^{-} \mathrm{LDH}(x=0.33)$ cannot be satisfactorily accounted for by any of the polytypes predicted by Bookin and Drits or by their intergrowths.

We therefore ask the questions:

1. Does the Bookin and Drits approach, relying as it does on the close packing of hydroxyl ions, predict the complete universe of polytypes possible in the LDH system?

2 . Is there a more general approach to the prediction of polytypes that expands on the universe of Bookin and Drits and predicts other polytypes whose stacking sequences depart from the pattern of close packing of hydroxyl ions?

In this paper, we address these questions and offer a different and a more general approach to the prediction of polytypes based on symmetry considerations.

\section{Discussion}

\section{Structural Synthon Approach to Predicting the Polytypes}

In an earlier paper, ${ }^{[14]}$ we described a 'structural synthon' as a certain packing of atoms, which like its better known mo- lecular analogue, the 'supramolecular synthon', ${ }^{[20]}$ repeats itself in a very diverse range of solids. The AC layer with the generalized composition $\left[\mathrm{MX}_{2}\right]$ is found in a very diverse range of materials such as metal sulfides $\mathrm{MS}_{2}(\mathrm{M}=\mathrm{Ti}$, Mo, $\mathrm{Nb}, \mathrm{Ta}),{ }^{[21,22]}$ metal selenides $\mathrm{MSe}_{2}(\mathrm{M}=\mathrm{Mo}, \mathrm{Ta}),{ }^{[23]}$ divalent halides $\mathrm{MX}_{2}(\mathrm{M}=\mathrm{Ca}, \mathrm{Mg}, \mathrm{Fe}, \mathrm{Co}, \mathrm{Ni}, \mathrm{Cd}),{ }^{[24,25]}$ metal hydroxides $\mathrm{M}(\mathrm{OH})_{2}(\mathrm{M}=\mathrm{Mg}, \mathrm{Ni}, \mathrm{Co}),{ }^{[26,27]}$ birnessite-type oxides $\mathrm{MO}_{2}(\mathrm{M}=\mathrm{Mn}),{ }^{[28,29]}$ lithium oxides $\mathrm{LiMO}_{2}{ }^{[30,31]}$ and basic salts $\mathrm{M}(\mathrm{OH})_{2-x} \mathrm{~A}_{x}(\mathrm{M}=\mathrm{Ni}, \mathrm{Co} ; x=0.66-0.25 ; \mathrm{A}=\mathrm{Cl}$, $\left.\mathrm{NO}_{3}\right) .{ }^{[32-34]}$ We therefore call the AC layer a 'structural synthon', the term "structural" is used to describe the extended nature of the AC layer, as opposed to the sub.-molecular synthon originally proposed by Corey ${ }^{[35]}$ and the supramolecular synthon of Desiraju ${ }^{[36,37]}$ which are entities of finite dimension. In this paper, apart from the robust ubiquity we further propose that the structural synthon is characterized by its symmetry.

A typical AC layer (Figure 1) has the following symmetry elements:

(i) 3 and -3 axes normal to the layer and parallel to the stacking direction present at $(0,0),(1 / 3,2 / 3)$ and $(2 / 3,1 / 3)$,

(ii) 2 axes parallel to the layer and

(iii) mirror planes normal to the layer.

The layer group is $P \overline{3} 2 / m 1$.

This layer also constitutes the basic building block of all the LDHs. The various polytypes of LDHs are derived by stacking of the AC layers one above another. We describe below, the various possible stacking sequences guided by symmetry criteria.

\section{Stacking Sequences that Conserve Symmetry Elements Perpendicular to Layers}

As illustrated in Figure 1, 3-fold axes perpendicular to the layer are present at $(0,0),(1 / 3,2 / 3)$ and $(2 / 3,1 / 3)$. Hence the obvious ways of stacking layers to conserve 3 -fold symmetry is by translating them with respect to one another using the stacking vectors (also called 'layer displacements'), (i) $(0,0)$, (ii) $(1 / 3,2 / 3)$, (iii) $(2 / 3,1 / 3)$ or their combinations. Yet another way of conserving the 3 -fold axis is to stack the layer $\mathrm{AC}$ and

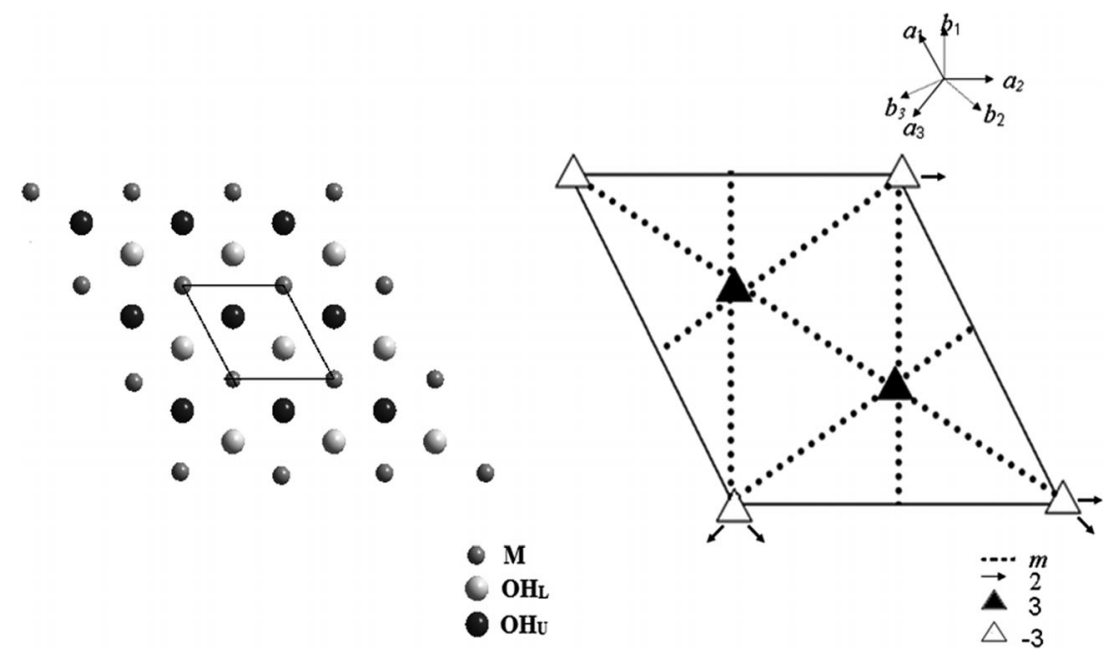

Figure 1. (a) Structure of a single layer viewed down the $c$-axis, (b) schematic of the symmetry elements present in a single layer. 
its mirror image CA one above the other alternatively by any of the stacking vectors mentioned above. This would generate 2-layered polytypes. However in doing so, the 3-fold axes of the adjacent layers are related by a mirror and are transformed into a $6_{3}$ screw.

All the polytypes derived by Bookin and Drits ${ }^{[8]}$ can be envisaged as stacking of layers using one of these stacking vectors. For instance stacking the AC layers exactly one above the other i.e., using $(0,0, z)(z=$ interlayer distance) stacking vector results in a structure having a single layered unit cell with hexagonal symmetry which is conventionally designated as ' $1 \mathrm{H}$ '. Such an arrangement of layers leads to a staggered arrangement of lower hydroxyl groups of the second layer with the upper hydroxyl groups of the first layer defining an octahedral interlayer site (Figure 2a). The PXRD pattern corresponding to such a polytype along with the appropriate indices is given in Figure 3.

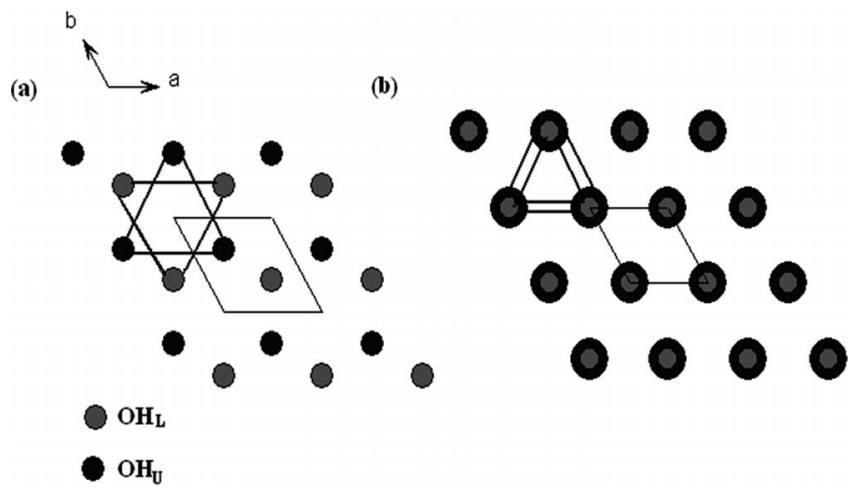

Figure 2. Schematic of the interlayer sites having (a) prismatic and (b) octahedral symmetry (L: Lower hydroxyl of the upper layer; U: Upper hydroxyl of the lower layer).

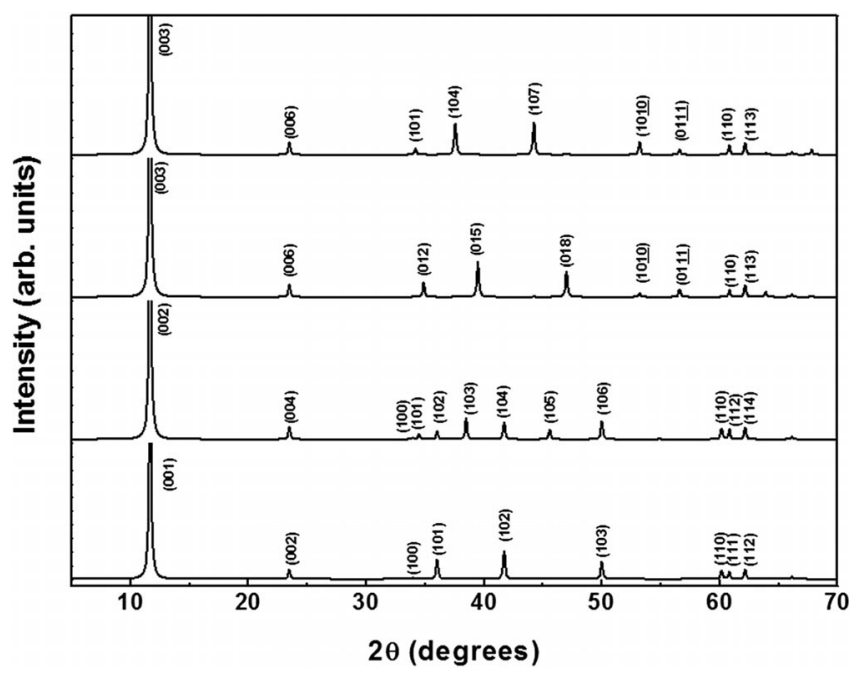

Figure 3. DIFFaX simulation of the PXRD patterns corresponding to $1 \mathrm{H}, 2 \mathrm{H}_{1}, 3 \mathrm{R}_{1}$ and $3 \mathrm{R}_{2}$ polytypes.

The two layered polytypes derived by Bookin and Drits can be envisaged as stacking of $\mathrm{AC}$ and $\mathrm{CA}$ layers using the stacking vectors $(0,0),(1 / 3,2 / 3),(2 / 3,1 / 3)$ or a combination of these. The stacking vector $(0,0, \mathrm{z})$ generates a two layered hexagonal cell, $\mathrm{AC}=\mathrm{CA}$, with eclipsed arrangement of the hydroxyl groups of the adjacent layers generating prismatic interlayer sites (Figure 2b). This structure is analogous to arrangement of layers in $2 \mathrm{H}_{1}$ polytype $(\mathrm{AC}=\mathrm{CA}=\mathrm{AC}-)$ derived by Bookin and Drits. The stacking vector $(1 / 3,2 / 3, z)$ generates a cell $\mathrm{AC}-\mathrm{AB}$ found in the $2 \mathrm{H}_{2}$ polytype of Bookin and Drits having octahedral interlayer sites. The stacking vector $(2 / 3$, $1 / 3, z$ ) also generates the $2 \mathrm{H}_{2}$ polytype with an enantiomeric stacking sequence $\mathrm{AC}-\mathrm{BC}-\mathrm{AC}-$. The sequence $\mathrm{AC}-\mathrm{BA}=\mathrm{AC}-$ , the $2 \mathrm{H}_{3}$ polytype of Bookin and Drits, is generated by stacking the $\mathrm{AC}$ layers in such a way that alternative layers are translated by the stacking vector $(1 / 3,2 / 3, z)$ with respect to AC. The interlayer sites generated in this case are alternatively octahedral and prismatic.

For higher layer periodicities the number of possible ways of stacking the layers increases generating a larger family of polytypes. In the case of 3-layered unit cells, the layers can be stacked in nine different ways out of which two are of rhombohedral symmetry and seven are of hexagonal symmetry.

Stacking of successive layers by $(2 / 3,1 / 3, z)$ leads to a three layered rhombohedral cell with prismatic interlayer site and is analogous to the $3 \mathrm{R}_{1}$ polytype with a stacking sequence $\mathrm{AC}=\mathrm{CB}=\mathrm{BA}=\mathrm{AC}-$. On the other hand stacking layers by $(1 / 3$, $2 / 3, z$ ) stacking vector generates a 3-layered rhombohedral cell with exclusively octahedral interlayer sites with a stacking sequence $\mathrm{AC}-\mathrm{BA}-\mathrm{CB}-\mathrm{AC}-$ the $3 \mathrm{R}_{2}$ polytype of Bookin and Drits nomenclature. ${ }^{[8]}$

Similarly, all the 3-layered hexagonal polytypes can be derived in this way by translating the layer with one of the stacking vectors described earlier or by a combination of reflection and translation. Seven different possibilities arise out of which two of them have exclusively octahedral interlayer sites and five comprise both kinds of interlayer sites.

Polytypes having 6-layer periodicities can be derived in a similar fashion. All of them can be envisaged as stacking of three 2-layered polytypes. While some of them exclusively comprise either prismatic or octahedral interlayer sites, most of them have a mixture of both.

In all these cases, diffraction patterns corresponding to different polytypes were simulated using the program $\operatorname{DIFFaX}^{[38,39]}$ (see Figure 3 for a few select simulations) and the Laue symmetry computed (Table 1 ). The $2 \mathrm{H}$ polytypes yield the Laue symmetry $6 / \mathrm{mmm}$, while the $1 \mathrm{H}$ and $3 \mathrm{R}$ polytypes yield $\overline{3} m$. The local symmetry of the interlayer sites, defined by the two closest set of hydroxyl oxygen atoms belonging to adjacent layers, was computed using the program Symgroup, ${ }^{[40]}$ and this is also listed in Table 1.

\section{Stacking Sequences that Destroy the 3-Axis and Conserve the 2-Axis}

Any translation other than the ones already described, destroys the 3-fold axis and reduces the symmetry of the resultant structure. As an illustration, we choose translations along the $a$ (or $b$ )-axis. Such a translation retains the two-fold axis parallel to the layer and one of the mirrors perpendicular to the layer and the resultant structure has monoclinic symmetry. Different 
Table 1. Stacking vectors which conserve the 3-fold symmetry of the $\left[\mathrm{MX}_{2}\right]$ synthon, the corresponding Laue symmetries of the crystal and the local symmetry of the interlayer site.

\begin{tabular}{llll}
\hline Polytype & Stacking vector & Laue Symmetry & Interlayer site symmetry \\
\hline $1 \mathrm{H}$ & $(0,0,1)$ & $\overline{3} m$ & $D_{3 d}$ \\
$2 \mathrm{H}_{1}$ & Reflection followed by $(0,0,1)$ & $6 / m m m$ & $D_{3 h}$ \\
$2 \mathrm{H}_{2}$ & Reflection followed by $(1 / 3,2 / 3) /(2 /$ & $6 / m m m$ & $D_{3 d}$ \\
& $3,1 / 3)$ & $\overline{3} m$ & $D_{3 h}$ \\
$3 \mathrm{R}_{1}$ & $(2 / 3,1 / 3)$ & $\overline{3} m$ & $D_{3 d}$ \\
$3 \mathrm{R}_{2}$ & $(1 / 3,2 / 3)$ & & \\
\hline
\end{tabular}
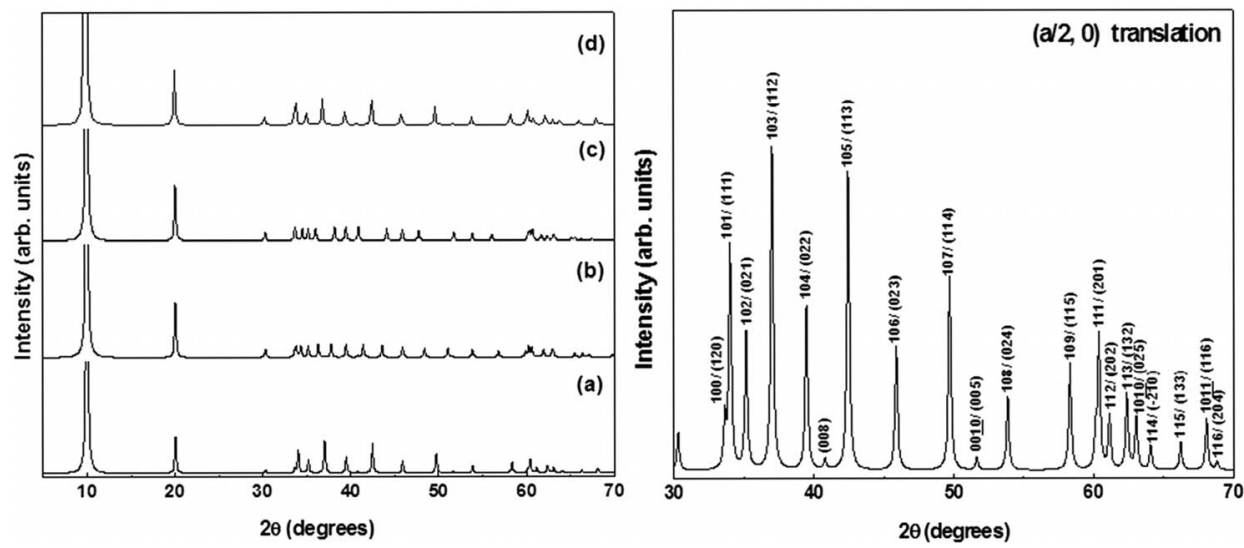

Figure 4. DIFFaX simulation of PXRD patterns of crystals with layer displacements (a) $(a / 2,0),(b)(a / 3,0),(c)(a / 4,0)$ and (d) $(a / 2, b / 2)$. On the right panel is given the detail of the $30-70{ }^{\circ} 2 \theta$ region of PXRD pattern at (a) (Indices given in parentheses correspond to monoclinic symmetry).

Table 2. Stacking vectors used to stack the $\left[\mathrm{MX}_{2}\right]$ synthon by displacements along $a$, the corresponding Laue symmetry of the crystal and symmetry of the interlayer site.

\begin{tabular}{lll}
\hline Stacking vector & Laue Symmetry generated by DIFFaX & Local symmetry of interlayer site \\
\hline$(a / 2,0, z)$ & $2 / m$ & $C_{2 v}$ \\
$(a / 3,0, z)$ & $\overline{1}$ & $C_{2 v}$ \\
$(a / 4,0, z)$ & $\overline{1}$ & $C_{2 v}$ \\
$(a / 2, b / 2, z)$ & & \\
\hline
\end{tabular}

stacking vectors $(a / n, 0, z)(n=2-5)$ were used. The PXRD patterns and the Laue symmetries of the resultant crystals were computed using DIFFaX (Figure 4, Table 2). While $(a / 2,0, z)$ translation generates a Laue symmetry $2 / m$, other translations generate $\overline{1}$. We designate the polytype generated by the $(a / 2$, $0, z$ ) translation as polytype ' $1 \mathrm{M}$ '. 1 stands for a single layered unit cell and $\mathrm{M}$ for monoclinic symmetry.

Figure 5 shows the schematic of two adjacent layers translated by $(a / 2,0)$ relative to one another generated using the structure visualization program Diamond. The corresponding interlayer site is no more prismatic. Symgroup predicts the local symmetry of the interlayer site to be $C_{2 v}$ (Table 2).

The Bragg peaks generated could be indexed to a single layered monoclinic unit cell with cell parameters, $a=3.067(8) \AA$, $b=5.324(1) \AA, c=8.977(2) \AA$ and $\beta=79.93(7)^{\circ}$. However the Bragg peaks generated by such a translation could also be indexed to a 2-layered hexagonal cell with a good figure of merit (Table 3). Further the apparent lowering of symmetry does not generate any new reflections, other than those predicted by the hexagonal cell. This raises the question: Is the

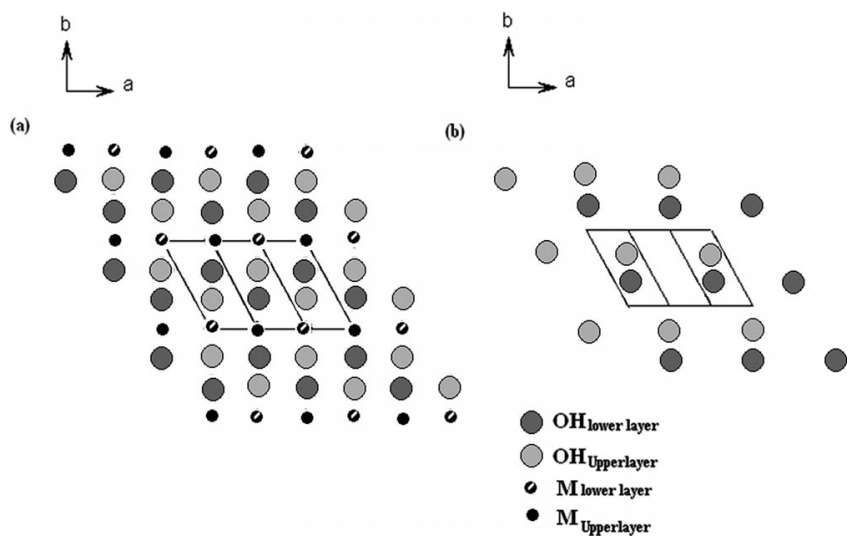

Figure 5. (a) Structure of two adjacent layers translated by the displacement vector $(a / 2,0)$ viewed down the normal to a-b plane and (b) schematic of the interlayer site corresponding to such a translation.

polytype generated by the $(a / 2,0, z)$ stacking vector just another polytype of hexagonal symmetry or a new polytype of monoclinic symmetry? 
Table 3. Calculated Bragg reflections of the $1 \mathrm{M}$ polytype and the corresponding indices within the hexagonal and monoclinic systems.

\begin{tabular}{|c|c|c|c|c|}
\hline $\begin{array}{l}\text { Calculated reflections for } \\
(a / 2,0, z) \text { translation } /^{\circ} 2 \theta\end{array}$ & $\begin{array}{l}2 \mathrm{H} \text { Cell } \\
a=b=3.075 \AA \\
c=17.6 \AA \\
\text { Figure of merit }=30.3 \\
2 \theta\end{array}$ & $h k l$ & $\begin{array}{l}\text { 1M Cell } \\
a=3.067 \AA ; \mathrm{b}=5.32 \AA \\
c=8.97 \AA ; \beta=79.93^{\circ} \\
\text { Figure of merit }=13.5 \\
2 \theta\end{array}$ & $h k l$ \\
\hline 10.09 & 10.0 & 002 & 10.0 & 001 \\
\hline 20.12 & 20.08 & 004 & 20.08 & 002 \\
\hline 30.32 & 30.33 & 006 & 30.32 & 003 \\
\hline 33.67 & 33.64 & 100 & 33.65 & 120 \\
\hline 34.09 & 34.03 & 101 & 34.09 & 111 \\
\hline 35.15 & 35.18 & 102 & 35.19 & 021 \\
\hline 37.06 & 37.03 & 103 & 37.05 & 112 \\
\hline 39.49 & 39.49 & 104 & 39.50 & 022 \\
\hline 40.79 & 40.83 & 008 & 40.81 & 004 \\
\hline 42.45 & 42.48 & 105 & 42.46 & 113 \\
\hline 45.94 & 45.92 & 106 & 45.93 & 023 \\
\hline 49.76 & 49.74 & 107 & 49.71 & 114 \\
\hline 51.70 & 51.69 & 0010 & 51.70 & 005 \\
\hline 53.87 & 53.89 & 108 & 53.89 & 124 \\
\hline 58.34 & 58.36 & 109 & 58.30 & 115 \\
\hline 60.37 & 60.40 & 111 & 60.34 & 201 \\
\hline 61.23 & 61.16 & 112 & 61.28 & 202 \\
\hline 62.44 & 62.40 & 113 & 62.42 & 132 \\
\hline 63.12 & 63.10 & 1010 & 63.12 & 025 \\
\hline 64.10 & 64.10 & 114 & 64.08 & -210 \\
\hline 66.29 & 66.15 & 115 & 66.28 & 133 \\
\hline 68.04 & 68.10 & 1011 & 68.06 & 116 \\
\hline 68.86 & 68.85 & 116 & 68.89 & 204 \\
\hline
\end{tabular}

We answer this question by relying on three factors:

(i) Given a choice of two cells, which predict all the observed Bragg reflections, convention favors the smaller of the two cells. Thereby a $1 \mathrm{M}$ cell is preferred to the $2 \mathrm{H}$.

(ii) A point symmetry test on each of the Bragg reflections reveals that the intensities of the $h 0 l$ and $0 h l$ reflections for hexagonal indexing are unequal. This indicates that the symmetry of the crystal obtained by stacking the AC layers by $(a / 2,0, z)$ stacking vector is no more hexagonal but is lowered to monoclinic symmetry.

(iii) The other possible explanation for the pattern getting indexed to hexagonal symmetry could arise due to the choice of the AC layer as the synthon. As the AC layer has a 3-fold symmetry, lateral displacement of these layers cannot generate new reflections in the $h-k$ plane, but only modulate the intensities of the observed reflections. ${ }^{[41]}$ The balance of judgment would tilt in favor of a single-layered monoclinic cell rather than a two-layered hexagonal cell based on intensity considerations.

\section{Experimentally Observed Polytypes}

Many of the polytypes envisaged by retaining 3-fold symmetry are observed either in the mineral form or by laboratory synthesis. Several authors have made a comprehensive review of the polytype diversity among this class of material. ${ }^{[10,42]} \mathrm{A}$ representative list of polytypes reported till date comprising different anions is given as Supporting Information SI. 1. It is evident that polytypes with large layer periodicities are rather uncommon. We ask the question: What are the factors respon- sible for the selection of a particular polytype in preference to the others?

Among the LDHs, contribution to the energy of a polytype arises from two chief factors: (a) bonding within the layer and (b) bonding between the layer and the interlayer. The metal hydroxide layer is the same across the polytypes and hence the contribution from the first coordination sphere to the total energy of different polytypes remains the same. Among the polytypes which conserve the 3 -fold symmetry, the second coordination sphere also remains the same. For a given anion, the contribution arising from the coulombic attraction between the layer and the interlayer to the total energy of different polytypes would also remain the same. Hence the total energy of different polytypes is comparable and laboratory synthesized LDHs often crystallize as intergrowths of two or more polytypes. However between LDHs containing different anions, the contribution of hydrogen bonding between the layer and the interlayer species to the total energy would vary greatly depending on the local symmetry of the interlayer sites. Hydrogen bonding is maximized when the molecular symmetry of the intercalated anion matches with the local symmetry of the interlayer site, a factor that helps polytype selection. ${ }^{[13,43]}$

All the predicted polytypes belonging to hexagonal symmetry are characterized by the presence of either octahedral (more specifically $D_{3 d}$ ) or prismatic interlayer sites or both. Carbonate containing LDHs crystallize in $3 \mathrm{R}_{1}$ or $2 \mathrm{H}_{1}$ structure as the molecular symmetry of $\mathrm{CO}_{3}{ }^{2-}\left(D_{3 h}\right)$ matches with the local symmetry of the prismatic interlayer sites. ${ }^{[43]}$ Sulfate containing LDHs are known to crystallize in variety of polytypic structures having either kinds of interlayer sites owing to 
the match in its coordination symmetry $\left(C_{3 v}\right)$ with both prismatic and octahedral interlayer sites. ${ }^{[13]}$ Sulfate-LDHs exhibit interpolytype transformations $(1 \mathrm{H} \leftrightarrow 3 \mathrm{R})$ at low temperatures indicating that the energy barrier between two polytypes for sulfate LDHs is low. ${ }^{[14]}$

In contrast with the carbonate ions, nitrate ions are known to coordinate in the $\mathrm{C}_{2 \mathrm{v}}$ mode, in the interlayer of LDHs with $x=0.33$ composition. ${ }^{[19]}$ In this mode of coordination, one of the NO bonds is parallel to the $c$-crystallographic axis. This coordination symmetry is incompatible with both prismatic and octahedral interlayer site symmetry. By Taylor's criterion $^{[43]}$ the nitrate-LDH is not expected to crystallize in either the hexagonal or rhombohedral symmetries predicted by Bookin and Drits. The expected crystal symmetry is monoclinic. The PXRD pattern of the nitrate-intercalated LDH $(x=$ 0.33 ) is shown in Figure 6. All the observed Bragg reflections correspond to a three layered rhombohedral polytype. Intensity motifs ( $h 0 l$ being weak and $0 \mathrm{kl}$ being strong) indicate that it belongs to $3 \mathrm{R}_{1}$ polytype. The broadening of the reflections in the mid. $2 \theta\left(30-55^{\circ}\right)$ region indicates the presence of structural disorder in the material. To account for the observed broadening we simulate the PXRD pattern corresponding to $3 \mathrm{R}_{1}$ polytype with $2 \mathrm{H}_{1}$ intergrowths (SI. 2), a disorder model that has been successfully used in the past and one which is in conformity with the Bookin and Drits approach. ${ }^{[17]}$ The fit obtained is poor $\left(R_{\mathrm{p}}=28.2\right)$ and in particular, sharp reflections expected at high angles $\left(60-70^{\circ} 2 \theta\right)$ are not observed. This indicates that a new disorder model is necessary to account for the observed pattern. We now introduce stacking fault motifs corresponding to the $1 \mathrm{M}$ polytype, envisaged in this work. The fit obtained is shown in Figure 6 and is of better quality $\left(R_{\mathrm{p}}=11.3\right)$. The match between the calculated and observed profiles at high angles is better than that in the earlier simulation shown in SI. 2. The DIFFaX technique is a simulation rather than a refinement and to the extent that the goodness of fit is improved, the new disorder model is superior to that employed earlier in the context of other LDHs.

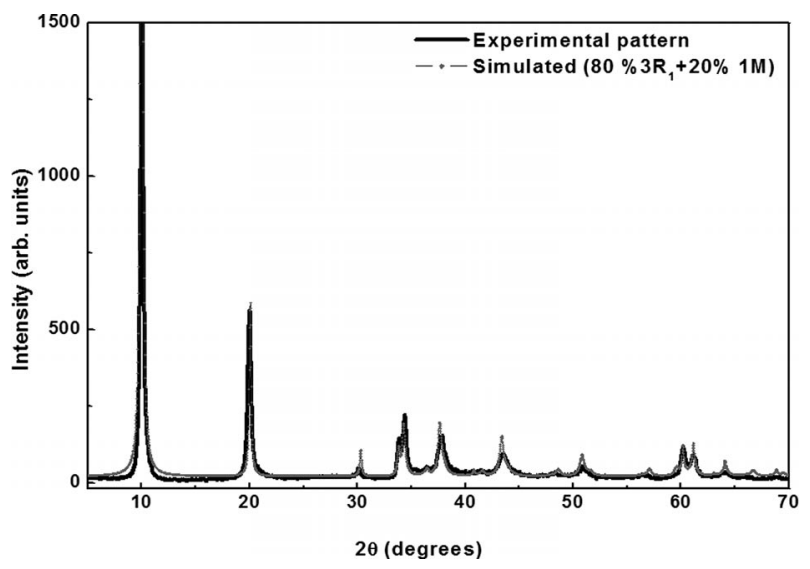

Figure 6. PXRD pattern of $\left[\mathrm{Zn}-\mathrm{Al}-\mathrm{NO}_{3}\right] \mathrm{LDH}$ overlaid with $\mathrm{DIFFaX}$ simulated pattern corresponding to $3 \mathrm{R}_{1}$ polytype with $1 \mathrm{M}$ faults.

However a pure monoclinic polytype based on the metal hydroxide layer belonging to the layer group $P \overline{3} 2 / m 1$ has not been reported till date and remains a legitimate challenge for synthesis efforts. Critical to the successful synthesis is the choice of an anion with a suitable molecular symmetry and appropriate precipitation conditions. Nitrate is a candidate anion to mediate the synthesis of a monoclinic polytype as it coordinates in the $C_{2 v}$ mode in the interlayer. However this work shows that it is not entirely successful as thermodynamic factors favor hexagonal polytypes, wherein the atoms are in a close packed arrangement. It is nevertheless interesting that we observe planar faults of monoclinic symmetry as a first step to the synthesis of an ordered polytype of monoclinic symmetry.

A monoclinic LDH based on a metal hydroxide layer of a different symmetry is reported by Krivovichev and coworkers $^{[44-46]}$ in the mineral quintinite. Here the monoclinic distortion arises due to cation ordering in the hydroxide layer. In this case the synthon itself differs from the one reported here and a separate analysis based on the formalism described in this work is necessary to predict the polytypes of cation ordered structures.

\section{Experimental Section}

The nitrate containing LDH was synthesized by co-precipitation by adding a mixed metal $(\mathrm{Zn}+\mathrm{Al})$ nitrate solution $([\mathrm{Zn}] /[\mathrm{Al}]=2)$ drop wise to a reaction vessel containing a $\mathrm{NaNO}_{3}$ salt solution $(100 \mathrm{~mL})$. The content of nitrate ions was 10 times in excess of the stoichiometric requirement. A constant $\mathrm{pH}$ of 8 was maintained during preparation by simultaneous addition of $0.5 \mathrm{M} \mathrm{NaOH}$ using a Metrohm model 718 STAT titrino operating in the $\mathrm{pH}$ STAT mode. The temperature was kept constant at $60{ }^{\circ} \mathrm{C}$ and nitrogen gas was bubbled continuously throughout the experiment. The resulting slurry was then aged for 15 $h$ and separated by centrifugation. The precipitate obtained was washed several times with decarbonated water and finally with acetone and dried at $60{ }^{\circ} \mathrm{C}$. Powder X-ray diffraction patterns of all the samples were obtained using a Bruker Model D8 Advance powder diffractometer $\left(\mathrm{Cu}-K_{\alpha}\right.$ radiation, $\lambda=1.541 \AA$, operating voltage $40 \mathrm{kV}$, current $30 \mathrm{~mA}$, scan rate $0.1^{\circ} 2 \theta \mathrm{min}^{-1}$ ).

\section{Computational Studies}

The diffraction patterns were simulated using the DIFFaX (Version 1.807), ${ }^{[38,39]}$ a FORTRAN based computer program. Within the DIFFaX formalism, a solid is treated as a stacking of layers of atoms and the PXRD pattern is computed by integrating the diffraction intensities generated layer by layer. Model simulations were performed using a single layer extracted from a published structure model (CCDC-86655) excluding the interlayer atoms. Patterns corresponding to different polytypes were generated by stacking the layers with corresponding stacking vectors with different probabilities. While calculating the diffraction pattern for different polytypes the symmetry was declared as unknown and the program was allowed to compute the Laue symmetry based on the generated intensities. PXRD patterns were indexed using the program PROZSKI ${ }^{[47]}$ and the figure of merits was determined. Structure viewing program Diamond (Version 3.0) ${ }^{[48]}$ was used to visualize the structure and the translation of layers with different stacking vectors. Symgroup, ${ }^{[40]}$ a Linux based program was used to calculate the local symmetry of the interlayer sites among different polytypes. For this Cartesian coordinates corresponding to the two set of closest hydroxyl groups belonging to adjacent layers were used as input in the program and the symmetry measures for different symmetry elements were computed. Interlayer site symmetry was de- 
rived based on the presence or absence of set of symmetry elements. Simulations of the observed PXRD patterns in SI. 1 and Figure 6 were performed including interlayer atoms by adapting the published structure of the sulfate-LDH (CCDC-91859).

Supporting Information (see footnote on the first page of this article): A Table of polytypes with different anions and the corresponding citations; DIFFaX simulation of the PXRD pattern of the [Zn-Al-NO3-] LDH including planar faults of hexagonal symmetry.

\section{Acknowledgments}

The authors thank Prof. M. M. J. Treacy for useful discussions. Authors thank the Department of Science and Technology (DST), Government of India (GOI) for financial support. SR is grateful for the award of Senior Research Fellowship of the Centre for Scientific and Industrial Research, GOI. PVK is a recipient of the Ramanna Fellowship of the DST.

\section{References}

[1] H. Baumhauer, Z. Kristallogr. 1912, 50, 33.

[2] A. R. Verma, P. Krishna, Polymorphism and Polytypism in Crystals, John Wiley \& Sons, New York, USA, 1966.

[3] D. G. Evans, X. Duan, Chem. Commun. 2006, 485.

[4] J. H. Choy, S. Y. Kwak, J. S. Park, Y. J. Jeong, J. Portier, J. Am. Chem. Soc. 1999, 121, 1399.

[5] F. Cavani, F. Trifiro, A. Vaccari, Catal. Today 1991,11, 173.

[6] S. V. Prasanna, P. V. Kamath, Solid State Sci. 2008, 10, 260.

[7] H. R. Oswald, R. Asper, Preparation and Crystal growth of Materials with Layered Structures, D. Riedel Publishing Company, 1977, 71.

[8] A. S. Bookin, V. A. Drits, Clays Clay Miner. 1993, 41, 551.

[9] H. P. Boehm, J. Steinle, C. Vieweger, Angew. Chem. Int. Ed. Engl. 1977, 16, 265.

[10] V. A. Drits, A. S. Bookin, Crystal Structure and X-ray Identification of Layered Double Hydroxides, In: Layered Double Hydroxides: Present and Future (Ed.: V. Rives), Nova Science: New York, USA, 2001, p. 39.

[11] C. Frondel, Amer. Miner. 1941, 26, 295

[12] A. S. Bookin, V. A. Drits, Clays Clay Miner. 1993, 41, 558

[13] S. Radha, P. V. Kamath, Cryst. Growth Des. 2009, 9, 3203

[14] S. Radha, J. C. A. Antonyraj, P. V. Kamath, S. Kannan, Z. Anorg. Allg. Chem. 2010, 636, 2658

[15] M. Bellotto, B. Rebours, O. Clause, J. Lynch, D. Bazin, E. Elkaim, J. Phys. Chem. 1996, 100, 8527

[16] G. S. Thomas, M. Rajamathi, P. V. Kamath, Clays Clay Miner. 2004, 52, 693

[17] A. V. Radha, C. Shivakumara, P. V. Kamath, Clays Clay Miner. 2005, 53, 521

[18] S. V. Prasanna, P. V. Kamath, Solid State Sci. 2008, 10, 260

[19] S. L. Wang, P. C. Wang, Coll. Surf. A 2007, 292, 131
[20] G. R. Desiraju, Angew. Chem. Int. Ed. Engl. 1995, 34, 2311

[21] F. Jellinek, G. Brauer, H. Müller, Nature 1960, 185, 376

[22] R. G. Dickinson, L. Pauling, J. Am. Chem. Soc. 1923, 45, 1923

[23] B. E. Brown, D. J. Beerntsen, Acta Crystallogr. 1965, 18, 31

[24] P. Day, A. Dinsdale, E. R. Krausz, D. J. Robbins, J. Phys. C 1976, 9, 2481

[25] Y. H. Liu, Y. M. Ma, Z. He, T. Cui, B. B. Liu, G. T. Zou, J. Phys. Condens. Matter 2007, 19, 425225

[26] M. Rajamathi, P. V. Kamath, R. Seshadri, J. Mater. Chem. 2000, 10,503

[27] C. Delmas, C. Tessier, J. Mater. Chem. 1997, 7, 1439

[28] A. C. Gaillot, D. Flot, V. A. Drits, A. Manceau, M. Burghammer, B. Lanson, Chem. Mater. 2003, 15, 4666

[29] V. A. Drits, B. Lanson, A. C. Gaillot, Amer. Miner. 2007, 92, 771

[30] Y. S. Horn, S. A. Hackney, J. Solid State Chem. 1998, 140, 116

[31] G. G. Amatucci, J. M. Tarascon, L. C. Klein, J. Electrochem. Soc. 1996, 143,1114

[32] P. B. Rocherulle, J. Rius, D. Louer, J. Solid State Chem. 1997, 128,295

[33] M. Rajamathi, P. V. Kamath, Mater. Lett. 2003, 57, 2390

[34] T. N. Ramesh, M. Rajamathi, P. V. Kamath, J. Solid State Chem. 2006, 179,2386

[35] E. J. Corey, Pure Appl. Chem. 1967, 14, 19

[36] J. A. R. P. Sarma, G. R. Desiraju, Cryst. Growth Des. 2002, 2, 93

[37] D. S. Reddy, Y. E. Ovchinnikov, O. V. Shishkin, Y. T. Struchkov, G. R. Desiraju, J. Am. Chem. Soc. 1996, 118, 4085

[38] M. M. J. Treacy, M. W. Deem, J. M. Newsam, Computer Code DIFFaX, Version 1.807, NEC Research Institute, Inc., Princeton, New Jersey, USA, 2000.

[39] M. M. J. Treacy, J. M. Newsam, M. W. Deem, Proc. R. Soc. London 1991, A433, 499

[40] D. Casanova, P. Alemany, S. Alvarez, SYMOP, Program for the Calculation of Continuous Symmetry Operation Measures, Version 1.0, Universidad de Barcelona, Barcelona, Spain, 2007.

[41] M. M. J. Treacy, Personal Communication, April 22, 2011.

[42] D. G. Evans, R. C. T. Slade, Structural Aspects of Layered Double Hydroxides, in: Layered Double Hydroxides (Eds: X. Duan, D. G. Evans), Springer, 2005.

[43] H. F. Taylor, Miner. Magn. 1973, 39, 377

[44] S. V. Krivovichev, V. N. Yakovenchuk, A. A. Zolotarev, G. N. Ivanyuk, Y. A. Pakhomovsky, Chimia 2010, 64, 1

[45] S. V. Krivovichev, V. N. Yakovenchuk, E. S. Zhitova, A. A. Zolotarev, Y. A. Pakhomovsky, G. N. Ivanyuk, Miner. Magn. 2010, 74, 833

[46] S. V. Krivovichev, V. N. Yakovenchuk, E. S. Zhitova, A. A. Zolotarev, Y. A. Pakhomovsky, G. N. Ivanyuk, Miner. Magn. 2010, 74, 841

[47] W. Lasocha, K. Lewiniski, PROSZKI, A System of Programs for Powder Diffraction Data Analysis, ver-2.4; Krakow, Poland. 1994.

[48] K. Brandenburg, H. Putz, DIAMOND, Crystal and Molecular Visualization Tool, Version 3.0, Crystal Impact GmbH, Bonn, Germany, 2004.

Received: June 04, 2012

Published Online: August 24, 2012 